\title{
Health Care Provider Knowledge and Practices Regarding Folic Acid, United States, 2002-2003
}

\author{
Jennifer L. Williams • Stephen M. Abelman • \\ Elizabeth M. Fassett • Cheryl E. Stone • \\ Joann R. Petrini · Karla Damus · Joseph Mulinare
}

Published online: 24 May 2006

(C) Springer Science+Business Media, Inc. 2006

\begin{abstract}
Objective: To assess health care providers (HCP) knowledge and practices regarding folic acid (FA) use for neural tube defect (NTD) prevention. Methods: Two identical surveys were conducted among 611 obstetricians/gynecologists (OB/GYNs) and family/general physicians (FAM/GENs) (2002), and 500 physician assistants (PAs), nurse practitioners (NPs), certified nurse midwives (CNMs), and registered nurses (2003) to ascertain knowledge and practices regarding FA. For analysis, $T$-tests, univariate and multivariate logistic regression modeling were used. Results: Universally, providers knew that FA prevents birth defects. Over $88 \%$ knew when a woman should start taking folic acid for the prevention of NTDs; and over $85 \%$ knew FA supplementation beyond what is available in the diet is necessary. However, only half knew that $50 \%$ of all pregnancies in the United States are unplanned. Women heard information about multivitamins or FA most often during well woman visits in obstetrical/gynecology (ob/gyn) practice settings $(65 \%)$, and about $50 \%$ of the time during well woman visits in family/general (fam/gen) practice settings and $50 \%$ of the time at gynecology visits (both settings). Among all providers, $42 \%$ did not know the correct FA dosage (400 $\mu \mathrm{g}$ daily). HCPs taking multivitamins were
\end{abstract}

J. L. Williams $(\bowtie) \cdot$ E. M. Fassett · J. Mulinare

National Center on Birth Defects and Developmental Disabilities, Centers for Disease Control and Prevention,

1600 Clifton Road, NE MS E 86, Atlanta, GA 30333 USA

e-mail: jwilliams2@cdc.gov

S. M. Abelman · J. R. Petrini $\cdot$ K. Damus

March of Dimes,

New York, NY, USA

C. E. Stone

Rynne Marketing,

Evanston, IL, USA more than twice as likely to recommend multivitamins to their patients (Odds Ratio [OR] 2.27 95\%, Confidence Interval [CI] 1.75-2.94). HCPs with lower income clients (OR 1.49, CI 1.22-1.81) and HCPs with practices having more than $10 \%$ minorities (OR 1.46, CI 1.11-1.92) were more likely to recommend supplements. NPs in ob/gyn settings were most likely and FAM/GENs were least likely to recommend supplements (OR 3.06, CL 1.36-6.90 and OR 0.64, CL $0.45-0.90$ respectively). Conclusions: Knowledge about birth defects and the necessity of supplemental FA was high. Increasing knowledge about unintended pregnancy rates and correct dosages of FA is needed. The strongest predictor for recommending the use of FA supplements was whether the provider took a multivitamin.

Keywords Health care provider - Folic acid .

Knowledge $\cdot$ Behavior

\section{Introduction}

Birth defects continue to be the leading cause of infant mortality in the United States [1], and those of the nervous system, including neural tube defects (NTDs), are the third leading type of birth defect [2]. NTDs are serious birth defects of the brain (anencephaly) and spine (spina bifida), affecting approximately 3000 pregnancies each year in the United States [3]. Studies show that $50-70 \%$ of these birth defects can be prevented if a woman consumes adequate folic acid (FA) daily before conception and throughout the first trimester of pregnancy [4, 5]. The U.S. Public Health Service (USPHS) (1992) and Institute of Medicine (IOM) (1998) issued separate recommendations that all women of childbearing age consume $400 \mu \mathrm{g}$ of FA daily for the prevention of NTDs [5, 6]. Women can obtain adequate amounts of FA by taking a folic acid 
supplement, taking a multivitamin containing folic acid, or by eating cereal grain products fortified with $100 \%$ of the RDA of folic acid.

In 1998, the U.S. Food and Drug Administration required mandatory fortification of enriched cereal grains with $140 \mu \mathrm{g}$ of FA per $100 \mathrm{~g}$ of cereal grain product in an effort to reduce the occurrence of NTDs [7]. It was estimated that FA fortification at this level would provide about $0.1 \mathrm{mg}$ of additional folic acid in the average diet. Recent evidence from the literature suggests that fortification has in fact doubled this initial projection [8]. Fortification of cereal grain products has resulted in a 27\% reduction in NTDs in the United States [3], which is relatively consistent with the observed population's increase of folic acid by $0.2 \mathrm{mg} / \mathrm{d}$ [9]. While fortification has made a significant contribution in preventing NTDs, most women in the U.S. still do not get enough FA from fortification alone. In a recent study examining serum folate levels of women of childbearing age post fortification, "less than $10 \%$ of women achieved a red blood cell folate level that is associated with a significant decrease of NTDs" through diet alone [10]. Therefore, it is still necessary for women of childbearing age to consume vitamins containing FA to get the daily recommended amount of FA (400 $\mu \mathrm{g})$.

Since 1995, the March of Dimes (MoD) and the Gallup Organization have conducted an annual national survey among women of childbearing age regarding their awareness, knowledge, and consumption of FA. Early results indicated a lack of awareness about FA among women of childbearing age. In 1999, in response to these results, the $\mathrm{MoD}$, the Centers for Disease Control and Prevention (CDC), and the National Council on Folic Acid (NCFA) began a concerted effort to increase FA awareness through education campaigns targeting women and health care providers (HCPs). Based on the MoD survey, FA awareness in women of childbearing age has risen from 52\% in 1995 to $77 \%$ in 2004 [11]. Lagging behind the increase in awareness, daily consumption of a multivitamin containing FA increased only slightly from 32\% in 1995 to $40 \%$ in 2004 [11, 12]. When asked what types of things would encourage them to take a multivitamin daily, unprompted, $42 \%$ of women who were aware of, but not consuming, FA said they would take a multivitamin if advised to do so by an HCP [11]. Additionally, in the 2004 HealthStyles Survey, 91\% of women 18 through 45 years of age agreed or strongly agreed that they would take a multivitamin daily to prevent birth defects during future pregnancies if their doctor encouraged them to do so (unpublished data) [13].

Two recent studies examined HCP practices regarding FA in the United States $[14,15]$. While providers were generally aware of folic acid's role in NTD prevention, only half of providers surveyed in both studies discussed folic acid on a regular basis with their patients of childbearing age.
In 2001, the MoD, in partnership with the CDC, initiated this project to examine HCP practices regarding FA. The objectives of this research are to:

- Assess HCP knowledge of FA recommendations, including dosage and timing for prevention of NTDs, among different provider types: obstetricians/gynecologists (OB/GYNs), family/general physicians (FAM/GENs), nurse practitioners (NPs), certified nurse midwives (CNMs), physician assistants (PAs), and registered nurses (RNs).

- Assess current practices among HCPs with respect to recommending FA to women of childbearing age.

- Identify the best means of supporting HCP efforts to promote FA consumption in female patients of childbearing age.

\section{Methods}

Random sample telephone surveys lasting an average of 12-18 minutes in length, were conducted in 2002 and 2003 among HCPs working in either obstetrical/gynecology (ob/gyn) practice settings or family/general (fam/gen) practice settings. These practice settings were selected because they represent the settings where the highest proportion of women of childbearing age receive care on a regular basis.

Geographic quotas were established to ensure a representation of respondents proportional to the actual distribution of office-based providers nationwide. Providers were identified through random sampling lists purchased from American Medical Information Inc., a division of InfoUSA. To identify sufficient representation of providers based in federally qualified health centers, a list of centers from the Health Resources and Services Administration's Bureau of Primary Health Care website (http://ask.hrsa.gov/pc/) was added to the sampling frame.

The identities of respondents were not revealed. This research met the requirements of Title 45 CFR part 46 for the protection of human subjects.

To encourage participation, respondents were offered a $\$ 50$ honorarium. The identity of the sponsors (CDC and $\mathrm{MoD}$ ) was not divulged to respondents. A cover letter from the research firm was faxed to participants who requested additional background information about the survey.

Health care providers were eligible to participate if they personally saw adult female patients for office or clinic visits, and worked in ob/gyn, or fam/gen medicine practices.

The surveys were presented as a series of true/false, multiple choice, and open-ended questions. The survey sought to determine the level of HCP knowledge regarding the role of FA in the prevention of NTDs, when HCPs recommended 
Table 1 Provider type and practice setting

\begin{tabular}{lcc}
\hline & \multicolumn{2}{l}{ Practice setting } \\
\cline { 2 - 3 } Provider type $^{a}$ & $\begin{array}{l}\text { Obstetrics/ } \\
\text { Gynecology }\end{array}$ & $\begin{array}{l}\text { Family/General } \\
\text { Medicine }\end{array}$ \\
\hline OB/GYN & 362 & 0 \\
FAM/GEN & 0 & 249 \\
PAs & 16 & 38 \\
NPs & 55 & 46 \\
CNMs & 200 & 0 \\
RNs & 77 & 63 \\
Total & 710 & 396 \\
\hline
\end{tabular}

Note. 5 missing provider variables not categorized.

${ }^{a}$ Provider type abbreviations: OB/GYN—obstetrician/gynecologist; FAM/GEN—family or general practice physician; PA—physician assistant; $\mathrm{NP}$ - nurse practitioner; $\mathrm{CNM}$ — certified nurse midwife; $\mathrm{RN}$ registered nurse.

FA, and how often HCPs recommended FA to women of childbearing age.

Data were analyzed using SPSS software (SPSS, 12.0, SPSS, Inc., 2003, Chicago, IL). $T$-tests, univariate comparisons and multivariate logistic regression modeling of the data were conducted. All analyses were tested for significance at the $95 \%$ confidence level ( $p$-value 0.05 ).

\section{Results}

Investigators surveyed 611 physician HCPs in 2002 and 500 nonphysician HCPs in 2003 (Table 1).

In 2002, 2552 physicians were screened as potentially qualified respondents. Of these 2552 physicians, 1213 (48\%) did not participate in surveys, $366(14 \%)$ requested additional information but did not schedule an interview, 184 (7\%) did not meet practice setting requirements, $142(6 \%)$ had fax numbers as the primary contact, $19(1 \%)$ were qualified refusals and $11(0.4 \%)$ had appointments set but not kept. The response rate for eligible physicians was $24 \%$.

In 2003, 650 nonphysician HCPs were screened as potentially qualified respondents. Of these 650 nonphysician HCPs, 73 (11\%) did not participate in surveys and $75(12 \%)$ were qualified refusals. The response rate for eligible nonphysician HCPs was $77 \%$.

The majority of physicians were male (77\%), younger than 55 years of age (69\%), and in practice over 20 years (50\%). Nonphysician HCPs were typically female (97\%) and younger than 55 years of age (82\%); $28 \%$ had been in practice over 20 years. Nonphysician HCPs were two times more likely than physician HCPs to work with underserved populations (Odds Ratio [OR] 1.97, Confidence Interval [CI] 1.54-2.53). Nonphysician HCPs saw more women of childbearing age in their practices $(67 \%)$ than physicans $(52 \%)$ ( $p$-value $<0.000)$.

The following results were obtained from a series of true/false questions.

In general, knowledge that women need a supplemental source of FA was high $(\sim 85 \%)$. Universally, HCPs in both practice settings (97\%) knew correctly that FA can prevent some birth defects. However, only $\sim 60 \%$ of all providers knew that most grain products in the United States are fortified with FA. In addition, there were significant differences in response to this question among provider types according to practice setting, ranging from $71.3 \%$ of RNs in ob/gyn settings to $44.7 \%$ of PAs in fam/gen settings ( $p$-value 0.030 ).

The following data were obtained from multiple choice questions. Providers in ob/gyn settings were not more knowledgeable than providers in fam/gen settings about when a woman should start taking FA for the prevention of NTDs (at least one month prior to conception through the first trimester of pregnancy) ( $88 \%$ vs. $89 \%$, respectively, $p$-value 0.094).

Because the majority of respondents knew the correct timing for a woman to start taking FA, providers were asked to identify what percentage of pregnancies they thought were unintended in the United States. Only about half of HCPs in both settings knew that approximately $50 \%$ of all pregnancies in the United States are unplanned [16]. The most knowledgeable group was NPs in ob/gyn settings (58.9\%), and the least knowledgeable group was RNs in ob/gyn settings $(35.0 \%)$ ( $p$-value 0.002$)$. Providers answering incorrectly were more likely to underestimate rather than overestimate the unintended pregnancy rate. Although a woman's receptiveness to the FA message might increase during a preconception care visit, HCPs providing prenatal care saw only a quarter of their pregnant patients for a preconception care visit.

Over half $(58 \%)$ of all providers were knowledgeable about the amount of FA $(400 \mu \mathrm{g})$ a woman needs daily for prevention of NTDs. Providers in ob/gyn settings were more knowledgeable than providers in fam/gen settings. The most knowledgeable group was CNMs, with $73.4 \%$ knowing the correct daily dosage of FA; the least knowledgeable group was FAM/GEN physicians $(41.9 \%)$ ( $p$-value $<0.000)$. When asked about the amount of FA a woman with a prior NTD affected pregnancy should take to prevent the recurrence of a NTD (4 mg), overall knowledge dropped dramatically, with only about $30 \%$ of all providers knowing the correct dose. Providers in ob/gyn settings were more knowledgeable than providers in fam/gen settings, The most knowledgeable group was OB/GYNs $(46 \%)$ and the least knowledgeable were RNs in fam/gen settings $(11.1 \%)$ ( $p$-value $<0.000)$.

Of critical importance to increasing consumption of FA among women of childbearing age is whether FA is incorporated into preventive health care messages during 
provider/patient encounters. Providers were asked if they always, usually, sometimes, or never addressed multivitamins or FA during well-woman examinations. More providers in ob/gyn settings than in fam/gen settings stated they regularly (always/usually) addressed multivitamins or FA during wellwoman visits (65\% vs. $50 \%$, respectively). Women were 1.8 times more likely to hear about multivitamins or FA during encounters in ob/gyn settings than in fam/gen settings (CI 1.42-2.34). The group most likely to talk to patients about multivitamins and FA was NPs in ob/gyn settings (83.9\%); the least likely were FAM/GEN physicians $(45.6 \%$ ) ( $p$-value $<0.00)$. Fewer counseling opportunities were taken at other types of examinations. Women heard the FA message about half the time from providers in both settings at routine gynecology visits. As expected, the fewest counseling opportunities taken were at episodic visits in both settings.

Predictors for recommending a multivitamin at a wellwoman visit were determined. The type of provider and clinic setting variables were combined as the base variable, with $\mathrm{OB} / \mathrm{GYNs}$ as the referent group. The dependant variable was whether the HCP always or usually recommended a multivitamin during a well-woman visit. Variables examined were number of weekly visits, provider age, sex, personal vitamin use, provider's years in practice, ethnicity of patients, practice setting, socioeconomic status of patient base, patient age, clinic setting (urban, suburban, or rural) and region of practice. Clinic setting (urban, suburban, or rural), provider age, region of practice, provider's years in practice, number of weekly visits, and patient age variables were not statistically significant in univariate analysis.

Table 2 represents the variables included in the logistic regression model. In comparison with OB/GYNs, FAM/GEN physicians were statistically less likely (OR 0.64, CI $0.45-$ 0.90 ) to recommend multivitamins, while NPs in ob/gyn settings were three times more likely (OR 3.06, CI 1.366.90) than their OB/GYN physician counterparts to provide counsel on multivitamins. While not quite reaching significance, CNMs were 1.6 times more likely (OR 1.56 , CI 0.96-2.53) than OB/GYNs to provide counsel on multivitamins. All other groups were not significantly different from OB/GYNs. Interestingly, whether a provider personally took a multivitamin was one of the strongest predictors of recommending multivitamin use to patients. Providers who regularly took a multivitamin were 2.3 times more likely (OR 2.27, CI 1.75-2.94) to recommend multivitamin use to their patients. Additional predictors of recommending multivitamin use were whether a provider stated that his or her practice's patient base was classified as poverty or low income, or if the practice consisted of at least $10 \%$ minorities. Being a female provider was also correlated with recommending multivitamin use to women of child-bearing age.

Approximately three-quarters of all respondents had seen information about FA in the last year. Unprompted, both
Table 2 Factors associated with recommending multivitamins at well-woman visits included in multivariate logistic regression model

\begin{tabular}{|c|c|c|}
\hline Variables & Odds ratio & $\begin{array}{l}95 \% \text { Confidence } \\
\text { interval }\end{array}$ \\
\hline \multicolumn{3}{|l|}{ Provider Type } \\
\hline OB/GYN & Referent & Referent \\
\hline FAM/GEN & 0.64 & $0.45-0.90^{*}$ \\
\hline CNM & 1.56 & $0.96-2.53$ \\
\hline NP in ob/gyn setting & 3.06 & $1.36-6.90^{*}$ \\
\hline PA in ob/gyn setting & 0.89 & $0.30-2.66$ \\
\hline $\mathrm{RN}$ in ob/gyn setting & 0.80 & $0.45-1.43$ \\
\hline NP in fam prac setting & 0.90 & $0.45-1.79$ \\
\hline PA in fam prac setting & 0.63 & $0.30-1.34$ \\
\hline $\mathrm{RN}$ in fam prac setting & 0.92 & $0.48-1.77$ \\
\hline \multicolumn{3}{|l|}{ Gender } \\
\hline Male & Referent & Referent \\
\hline Female & 1.62 & $1.11-2.36^{*}$ \\
\hline \multicolumn{3}{|l|}{ Provider takes a multivitamin } \\
\hline No & Referent & Referent \\
\hline Yes & 2.27 & $1.75-2.94^{*}$ \\
\hline \multicolumn{3}{|l|}{ SES of patient base } \\
\hline Middle or high income & Referent & Referent \\
\hline Poverty or low income & 1.49 & $1.22-1.81^{*}$ \\
\hline \multicolumn{3}{|l|}{ Minorities } \\
\hline$<10 \%$ minorities & Referent & Referent \\
\hline$>10 \%$ minorities & 1.46 & $1.11-1.92^{*}$ \\
\hline
\end{tabular}

${ }^{*}$ Denotes statistical significance.

physician and nonphysician HCPs reported that the organization most likely to have provided that information was the $\mathrm{MoD}$ (25\% and $46 \%$ respectively). Medical journals were identified most frequently as a way to effectively reach providers with information about FA (66\% physicians, $62 \%$ nonphysicians). Nonphysician providers reported the Internet as the second most effective way (15\%), and physicians reported either medical associations or conferences as the second most effective way to reach them with information about FA (12 and 11\%, respectively). Providers identified up to two types of resources they would like to have to promote FA intake among patients. Printed materials were the most popular type of resource requested by HCPs. Sixty percent $(60 \%)$ of physician and $69 \%$ of nonphysician providers requested brochures to distribute to their patients. Additional print resource requests included charts and articles. Free vitamins for patients were also requested (data not shown).

\section{Discussion}

These findings suggest that physician and nonphysician providers in both ob/gyn and fam/gen settings know that FA can prevent NTDs and that FA must be used from at least one month before pregnancy and continued through the first trimester. Consistent with other studies, provider knowledge 
of FA did not necessarily translate into counseling patients about its benefits $[15,17]$.

Providers in ob/gyn settings were almost twice as likely as providers in fam/gen settings to regularly address multivitamin or FA use during well-woman visits. Although NPs and CNMs in ob/gyn settings were most likely to regularly address multivitamin or FA use with their patients, these providers might not have as many opportunities to counsel women who are not contemplating pregnancy. Because providers in fam/gen settings provide routine care to large numbers of women of childbearing age, they play an important role in counseling women about FA consumption. Healthy People 2010 calls for an increase in the number of pregnancies begun with an optimum FA level. Providers in fam/gen settings are critical participants in the process of making the goals of Healthy People 2010 a reality $[18,19]$. Greater opportunities for promoting FA exist through changing provider practice behavior in fam/gen settings. It also appears that well-woman examinations in fam/gen settings and routine gynecology visits in both settings are important yet underutilized opportunities for HCPs to counsel women about multivitamin and FA use. The episodic visit was the least utilized, but with all the competing priorities at these types of encounters, counseling of multivitamins might not take precedence over other topics to be covered within limited timeframes.

Providers in ob/gyn settings were more likely than providers in fam/gen settings to know the correct amount of FA needed for NTD prevention (400 $\mu \mathrm{g}$ daily). Less than a third of all providers knew the correct amount of FA needed for the prevention of recurrence $(4 \mathrm{mg})$. Because women with a previous NTD-affected pregnancy are at an increased risk for another NTD-affected pregnancy, it is important for all providers to know the correct amount of FA needed for recurrence prevention.

Only about half of providers in both practice settings knew that approximately $50 \%$ of pregnancies in the United States are unplanned. Without screening their female patients about pregnancy intention, perhaps providers should be recommending FA to all women of childbearing age.

One of the most interesting findings was that the providers surveyed "practice what they preach." Providers who regularly took multivitamins were more likely to recommend multivitamins to their patients. Studies looking at other health behaviors have shown this as well $[20,21]$. Thus, including personal health behavior change messages in HCP outreach about multivitamins and FA might be advantageous.

In this dataset, a practice that served predominantly poverty or low income (low socioeconomic status) clientele or a practice consisting of at least $10 \%$ minorities predicted recommending a multivitamin. Among minorities and women with low socio-economic status, critical gaps in knowledge and practice on the benefits of periconceptional folic acid have been documented, especially among Hispanics, who are most vulnerable for NTDs [11, 22-24]. The findings in this survey that minorities and women of lower socio-economic status do appear to be targeted is encouraging, and underscores the importance of preconception care education programs for healthcare providers serving highrisk populations.

This study has certain limitations. Physician HCPs had a low response rate compared to nonphysician HCPs. We cannot comment on the providers who did not respond to the survey. Providers answering the survey might differ in knowledge and practice than non-responding providers. Additionally, one provider group surveyed (PAs) had small numbers. Many of the variables examined were associated with one another. In the logistic regression modeling, female sex of the HCP was a significant factor in predicting recommending multivitamins to female patients of childbearing age. This is most likely because the majority of the providers surveyed in 2003 were women.

\section{Conclusions}

While it would be most beneficial for women of childbearing age to get adequate folic acid from fortified foods alone, alternatives include efforts to educate all childbearing aged women. Several approaches to educating and promoting folic acid consumption are endorsed by the U.S. Public Health Service. These approaches include direct to consumer marketing, public health awareness activities, health care provider outreach, and policy strategies. This study examined just one aspect (HCPs) of the multifaceted approach needed to increase FA intake among women of childbearing age. Collaboration between public health and medical care services is critical in educating women about FA and multivitamin use as health care providers are trusted professionals. Mobilizing folic acid awareness efforts to target HCPs might assist providers with patient preconception care education. Training providers while in school or residency programs, or by offering continuing education courses that cover methods of applied behavior change and health education might be useful for increasing the numbers of women hearing about folic acid from their health care provider. Additionally, further research on what barriers HCPs experience when providing preventive care, such as provider time constraints, self-efficacy, and what their perceptions are on the benefits of preventive care, is needed. As many preventive behavior and wellness messages exist, providers are forced to prioritize according to the length of and reason for patient visits. Finding a creative way to make FA counseling a priority is critical to making the FA message a consistent part of the patient/health care provider dialogue. 
Acknowledgements The authors would like to acknowledge Margaret Watkins, Kathryn Lyon Daniel, Christine Prue, and Jennita Reefhuis of the CDC for their technical assistance with this project. Special thanks to the HCPs who participated in the survey. Without their help, this work would not have been possible.

\section{References}

1. Matthers TJ, Menaker F, MacDorman MF. Infant mortality statistics from the 2000 period linked birth/infant death data set. National Vital Statistics Report; 50(12). Hyattsville, Maryland: National Center for Health Statistics, 2002.

2. Source: National Center for Health Statistics, 2002 period linked birth/infant death data. Prepared by March of Dimes Perinatal Data Center, 2005.

3. Centers for Disease Control and Prevention. Spina bifida and anencephaly before and after FA mandate-United States, 1995-1996 and 1999-2000. MMWR 2004;53(17):62-5.

4. MRC Vitamin Study Research group. Prevention of neural tube defects: results of the Medical Research Council Vitamin Study. Lancet 1991;338:131-7.

5. Centers for Disease Control and Prevention. Recommendations for the use of folic acid to reduce the number of cases of spina bifida and other neural tube defects. MMWR 1992;41(No. RR-14).

6. Institute of Medicine. Dietary reference intake: Folate, other B vitamins, and choline. Washington, DC: National Academy Press, 1998.

7. United States Food and Drug Administration. Food standards. Fed Regist 1996;61:8781-97.

8. Choumenkovitch SF, Selhub J, Wilson PW, Rader JI, Rosenberg $\mathrm{IH}$, Jacques PF. Folic acid intake from fortification in United States exceeds predictions. Nutr Epidemiol 2002;132:2792-8.

9. Wald NJ, Law MR, Morris JK, Wald DS. Quantifying the effect of folic acid. Lancet 2001;358:2069-73.

10. Dietrich M, Brown CJP, Block G. The effect of folate fortification of cereal-grain products on blood folate status, dietary folate intake, and dietary folate sources among adult nonsupplement users in the United States. J Am Coll Nutr 2005;24(4); 266-74.
11. March of Dimes Birth Defects Foundation. (2004, June). Folic acid and the prevention of birth defects: A national survey of prepregnancy awareness and behavior among women of childbearing age 1995-2004. White Plains, NY.

12. Centers for Disease Control and Prevention. Use of vitamins containing folic acid among women of childbearing age-United States, 2004. MMWR 2004;53:847-50.

13. Porter Novelli 2004 HealthStyles Survey (Unpublished data, Porter Novelli International, Washington, DC). The data are property of PN.

14. Power ML, Holzman GB, Schuklin J. Knowledge and clinical practice regarding folic acid among obstetrician-gynecologists. Obstet Gynecol 2000;(6 Pt. 1):895-8.

15. Hauser KW, Lilly CM, Frias JL. Florida health care providers' knowledge of folic acid for the prevention of neural tube defects. South Med J 2004;97(5):437-9.

16. Henshaw SK. Unintended pregnancy in the United States. Fam Plann Perspect 1998;30(1):24-9.

17. Centers for Disease Control and Prevention. Knowledge and use of folic acid by women of childbearing age: United States, 1995 and 1998. MMWR 1999;48:325-7.

18. Hixon AL, Chapman RW. Healthy people 2010: The role of family physicians in addressing health disparities. Am Fam Physician 2000;62(9):1971-2, 1975, 1978.

19. United States Department of Health and Human Services, Public Health Service. Healthy people 2010. Conf ed. Washington, DC: 2000.

20. Frank E, Rothenberg R, Lewis C, Belodoff B. Correlates of physicians' prevention-related practices. Arch Fam Med 2000;9:35967.

21. Wells K, Lewis C, Leake B, Ware J. Do physicians preach what they practice? JAMA 1984;252(20):2846-8.

22. Ahluwalia IB, Lyon-Daniel K. Are women with recent live births aware of the benefits of folic acid? MMWR 2001;50(RR06):1-14.

23. Perlow JH. Comparative use and knowledge of preconceptional folic acid among Spanish- and English-speaking patient populations in Phoenix and Yuma, Arizona. Am J Obstet Gynecol 2001;184(6):1263-6.

24. Rosenberg KD, Gelow JM, Sandoval AP. Pregnancy intendedness and the use of periconceptional folic acid. Pediatrics 2003;111(5):1142-5. 